\section{(C) OPEN ACCESS}

\title{
Assessment of the incorporation of CNV surveillance into gene panel next-generation sequencing testing for inherited retinal diseases
}

\author{
Jamie M Ellingford, ${ }_{1}^{1,2}$ Bradley Horn, ${ }^{1}$ Christopher Campbell, ${ }^{1}$ Gavin Arno, ${ }^{3}$ \\ Stephanie Barton, ${ }^{1}$ Catriona Tate, ${ }^{4}$ Sanjeev Bhaskar, ${ }^{1}$ Panagiotis I Sergouniotis, ${ }_{1}^{1}$ \\ Rachel L Taylor, ${ }^{1,2}$ Keren J Carss, ${ }^{5,6}$ Lucy F L Raymond, ${ }^{6,7}$ Michel Michaelides, ${ }^{3,8}$ \\ Simon C Ramsden, ${ }^{1}$ Andrew R Webster, ${ }^{3,8}$ Graeme C M Black ${ }^{1,2}$
}

\begin{abstract}
- Additional material is published online only. To view please visit the journal online (http://dx.doi.org/10.1136/ jmedgenet-2017-104791).
\end{abstract}

For numbered affiliations see end of article.

\section{Correspondence to}

Graeme C M Black, Manchester Centre for Genomic Medicine, Manchester University NHS Foundation Trust, St Mary's Hospital, Manchester M13 9WL, UK; graeme.black@manchester. ac.uk

Received 10 May 2017 Revised 20 September 2017 Accepted 9 October 2017 Published Online First 26 October 2017
Check for updates

To cite: Ellingford JM, Horn B, Campbell C, et al. $J$ Med Genet

2018:55:114-121.

\section{ABSTRACT \\ Background Diagnostic use of gene panel next-} generation sequencing (NGS) techniques is commonplace for individuals with inherited retinal dystrophies (IRDs), a highly genetically heterogeneous group of disorders. However, these techniques have often failed to capture the complete spectrum of genomic variation causing IRD, including CNVs. This study assessed the applicability of introducing CNV surveillance into first-tier diagnostic gene panel NGS services for IRD.

Methods Three read-depth algorithms were applied to gene panel NGS data sets for 550 referred individuals, and informatics strategies used for quality assurance and CNV filtering. CNV events were confirmed and reported to referring clinicians through an accredited diagnostic laboratory.

Results We confirmed the presence of 33 deletions and 11 duplications, determining these findings to contribute to the confirmed or provisional molecular diagnosis of IRD for 25 individuals. We show that at least $7 \%$ of individuals referred for diagnostic testing for IRD have a CNV within genes relevant to their clinical diagnosis, and determined a positive predictive value of $79 \%$ for the employed CNV filtering techniques.

Conclusion Incorporation of CNV analysis increases diagnostic yield of gene panel NGS diagnostic tests for $I R D$, increases clarity in diagnostic reporting and expands the spectrum of known disease-causing mutations.

\section{INTRODUCTION}

Inherited retinal dystrophies (IRDs) are a set of genetic disorders that have a diverse pathogenesis and are characterised by extreme genetic and clinical heterogeneity. ${ }^{12}$ They are the leading cause of blindness in working-age adults in the $\mathrm{UK}^{3}{ }^{3}$ and are present in a range of multisystemic disorders, such as Usher syndrome and Senior-Loken syndrome. Identifying the genetic basis of IRDs can greatly assist the clinical diagnosis, counselling, treatment and management received by referred individuals. ${ }^{4}$ As a result, a number of genomic diagnostic tests are available for individuals with IRD, including SNP microarrays, direct sequencing approaches, array comparative genomic hybridisation (array CGH) and high-throughput sequencing (commonly referred to as next-generation sequencing, NGS). ${ }^{5}$
Despite the emergence of whole exome ${ }^{6}$ and whole genome NGS approaches, ${ }^{7}$ gene panel NGS approaches remain a major first-tier diagnostic test. This is due to their affordability, specificity, high coverage and proven capability to characterise disease-causing single nucleotide variations (SNVs) and small insertion and deletion events (indels). ${ }^{89}$ However, the informatics techniques used to detect genetic variation from gene panel NGS diagnostic services have often failed to truly characterise the spectrum of disease-causing variation within the IRDs, including the relative contribution of large structural variation and CNV.

$\mathrm{CNV}$ s result in the gain or loss of genomic material and are known to cause IRD. ${ }^{10}$ However, the insertion and breakpoints of CNVs are often deeply intronic or intergenic, and as a result are not captured by gene panel NGS approaches employed in diagnostic environments, which focus primarily on protein-coding regions and proven pathogenic intronic variants. This creates limitations in the types of variant detection algorithms that can be applied to gene panel NGS data sets to detect CNVs. ${ }^{11}$ Read-depth approaches for the surveillance of CNVs, with complementary quality assurance parameters, have recently been applied to gene panel NGS data sets in a diagnostic context. ${ }^{12-14}$ Moreover, recent studies investigating the role of CNVs in IRDs have identified an enrichment of disease-causing CNVs among individuals without a genetic diagnosis through gene panel NGS techniques, ${ }^{7} 15$ and demonstrated the capability of high-resolution array $\mathrm{CGH},{ }^{16}$ whole exome sequencing (WES) ${ }^{17}$ and whole genome sequencing (WGS) ${ }^{718}$ to identify CNVs within and encompassing these surveyed genes. While the potential to identify CNVs from gene panel NGS data sets for IRD has been shown, ${ }^{19}$ this analysis is yet to be extended to a large cohort of individuals using comprehensive NGS gene panels generated through accredited diagnostic services. As such, knowledge of the relative benefits and limitations of introducing CNV surveillance into first-tier diagnostic gene panel NGS services for IRD remains limited.

In this study, we have expanded the assessment of gene panel NGS diagnostic data sets to include CNV analysis among a large cohort of 550 individuals 
with IRD. Through comparison to WGS samples, we demonstrate the advantages and limitations of this approach, and illustrate an informatics workflow for the analysis of CNVs identified from gene panel NGS data sets. Taken together, incorporation of $\mathrm{CNV}$ analysis increases the diagnostic yield of a major first-tier diagnostic test for IRD, increases clarity in diagnostic reporting and expands the spectrum of known disease-causing mutations.

\section{MATERIALS AND METHODS}

\section{Recruitment of patients for CNV analysis}

We performed CNV analyses for 550 individuals with clinical indications of IRD. All individuals provided consent for the comprehensive analysis of variation in genes known as a cause of IRD and were referred for diagnostic genetic testing by clinicians at Manchester Royal Eye Hospital and Moorfields Eye Hospital, London.

\section{Generation of gene panel NGS data sets}

DNA was extracted from the peripheral blood of referred individuals and enriched for specified regions of the genome using an Agilent SureSelect Custom Design target-enrichment kit (Agilent, Santa Clara, California, USA). Enrichment kits were designed to capture known pathogenic intronic variants and the proteincoding regions \pm 50 nucleotides of selected National Center for Biotechnology Information (NCBI) RefSeq transcripts for 105 or 180 genes known as a cause of IRD (online supplementary table S1). Full details of the genes and analysis techniques used during the 105-gene diagnostic testing procedure (referred to as v2) can be found in Ellingford $e t a l^{9}$ and through the UK Genetic Testing Network (https://ukgtn.nhs.uk/find-a-test/search-by-disordergene/retinal-degeneration-105-gene-panel-568/). The 180-gene panel (referred to as v3) represents an expanded iteration of this diagnostic service within the UK National Health Service, with the additional inclusion of enrichment baits to capture (1) selected pathogenic intronic variants; and (2) additional genes known as a cause of IRD, including newly identified genes and genes known as a cause of congenital stationary night blindness. After enrichment, samples were pooled using unique barcode identifiers, and paired-end high-throughput sequencing was performed using the Illumina HiSeq 2000/2500.

\section{Detection of CNVs from gene panel NGS data sets using ExomeDepth}

Sequencing reads were demultiplexed with CASAVA V.1.8.2 and aligned to the $h g 19$ reference genome using Burrows-Wheeler Aligner short read (V.0.6.2) software. ${ }^{20}$ Duplicate reads were removed using SAMtools V.0.1.18 before variant calling was performed. We have described the methodology employed for the detection and clinical analysis of SNVs and indels previously. ${ }^{9} \mathrm{CNV}$ detection was performed using standard parameters for ExomeDepth V.1.1.6. ${ }^{21}$ ExomeDepth was presented with sets of aligned and non-duplicate sequencing reads in a binary sequence alignment/map (BAM) file format that were matched by gender and by the enrichment kit used, and had been generated for unrelated individuals with IRD referred for diagnostic testing (online supplementary table S2).

\section{Informatics filtering strategies}

We used three distinct strategies to limit the number of potential false-positive CNV events identified by ExomeDepth (figure 1). Events that were analysed in a clinical context were all (1) identified against three independent reference sets using ExomeDepth, (2) identified by at least one other CNV software tool (CoNVex, ${ }^{22}$

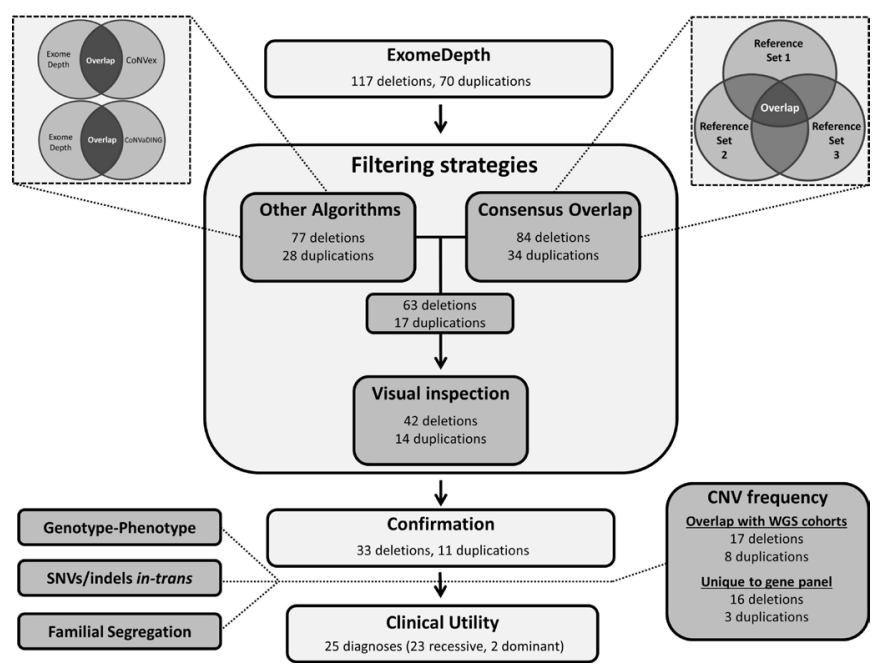

Figure 1 Informatics strategies used to filter CNVs identified by ExomeDepth. Taken together, these strategies had a positive predictive value of $79 \%$. SNV, single nucleotide variation; WGS, whole genome sequencing.

CoNVaDING $^{12}$ or both) and (3) visually inspected using the ExomeDepth graphical package.

We first limited our analysis of CNV events to those that had been identified by ExomeDepth in comparison to three mutually exclusive reference sets of samples. For each tested individual we created three randomly selected and non-overlapping groups of 30 individuals matched by their gender and the enrichment kit used and presented these to the ExomeDepth algorithm. The overlap between the three reference sets was calculated using bedtools V.2.25.0 intersect. Second, we performed CNV calling using two other publicly available CNV detection algorithms (CoNVex and CoNVaDING). Both algorithms were presented with aligned and non-duplicate sequencing reads in a BAM file format for large groups of individuals matched by gender and the enrichment kit used (as described in online supplementary table S2), and CNV calling was performed using standard parameters for each of these tools. We compared CNV events identified by CoNVex and CoNVaDING with those that had been identified by ExomeDepth using bedtools V.2.25.0 intersect, and included all events identified by ExomeDepth and at least one other CNV detection tool. We limited our third stage of analysis, visual inspection, to those events that were identified against three reference sets using ExomeDepth and by at least one additional $\mathrm{CNV}$ detection tool. Visual inspection included an assessment of the consistency of calculated read ratios across all exons within implicated genes, the extent of variation within the selected reference samples for each exon, the nature of the exon $\mathrm{CNV}$ status across the cohort and the continuity of abnormal CNV exons within the implicated gene.

\section{Clinical analysis of CNV events}

CNVs were interpreted alongside SNVs and indels that had been detected through routine gene panel NGS diagnostic techniques, as described previously. ${ }^{9}$ For each individual, variants were categorised in accordance with the American College of Medical Genetics and Genomics (ACMG) guidelines, ${ }^{23}$ and pathogenic/ likely pathogenic variants in a disease-causing state were determined to confirm or provisionally confirm a molecular diagnosis of IRD. CNV frequency estimations were calculated through comparison to 682 WGS data sets for individuals with clinical 
indications of IRD. Six hundred and five samples were generated using Illumina sequencing chemistry as part of the National Institute for Health Research (NIHR) BioResource Rare Diseases project, ${ }^{18}$ and the Manta and Canvas software algorithms were used to detect CNVs. ${ }^{24} 25$ Seventy-seven samples were generated using Complete Genomics sequencing chemistry, ${ }^{26}$ with CNVs identified using the Complete Genomics V.2.5 variant calling pipeline. ${ }^{27}$ Both of these strategies incorporate an assessment of sequencing read depth, an assessment of the read insert sizes and an assessment of sequencing read composition to identify $\mathrm{CNV}$ breakpoints/insertion points.

\section{Confirmation of identified CNVs}

CNVs were confirmed as present before they were reported to referring clinicians. Where kits designed and created by MRC-Holland (Amsterdam, The Netherlands) were available, we carried out multiplex ligation-dependent probe amplification (MLPA) assays. In the absence of a suitable MLPA kit, we validated CNVs using a digital droplet PCR or a quantitative fluorescence methodology, as described previously. ${ }^{14}$

\section{Estimating accuracy for CNV identification}

To ensure that the NGS data surveyed were appropriate for CNV surveillance, we calculated a series of sequencing coverage metrics. We have provided a full description of these calculated metrics and their utility previously, ${ }^{14}$ and these included (1) NGS coverage and normalised coverage for surveyed exons, (2) levels of insufficient coverage ( $<50$ unique NGS reads) for surveyed nucleotides and exons, and (3) intersample variability, defined as the coefficient of variation of normalised NGS coverage across samples selected as the reference set by ExomeDepth.

\section{RESULTS}

\section{CNV identification and filtering strategies}

We performed CNV calling for 550 individuals with IRD using gene panel NGS data sets generated through diagnostic testing in a clinically accredited laboratory (197v2 gene panel, 105 genes; 353 v3 gene panel, 180 genes). CNV surveillance was performed using ExomeDepth V.1.1.6. for four groups of individuals matched by their gender and the enrichment kit used during gene panel NGS (online supplementary table S2). In total, we identified 117 potential deletion events and 70 potential duplications through ExomeDepth (online supplementary table S3). This equated to an average of one CNV event per three individuals tested $(\min =0, \max =16)$, although we observed a trend of no CNVs identified for most samples $(n=429)$ and more than one CNV identified in few samples $(n=23$; online supplementary figure S1). We applied three distinct strategies for CNV filtering (see online supplementary methods and results) in order to identify true CNV events, and these analyses identified $56 \mathrm{CNV}$ events (30\% of the original 187) for further confirmation and clinical analysis (figure 1). To assess the accuracy of informatics filtering approaches, 13 events that were excluded through comparison to other CNV detection algorithms were also selected for further confirmation (online supplementary results).

\section{Estimating accuracy for CNV identification}

Through previous investigations we have identified that the level of NGS coverage in tested samples and the extent of variation in NGS coverage across selected reference samples (intersample variability) are both key influencers of the accuracy of ExomeDepth applied to gene panel NGS data sets. In total, we surveyed 1267742 exons for CNVs (1590 exons in 197 cases and 2704 exons in 353 cases), with an average of 2389 unique NGS reads generated per exon $(\min =0, \max =202357$, median $=1579, \mathrm{SD}=4013.7)$. We observed that $>50$ unique NGS reads were generated for all the nucleotides included within 99.2\% ( $n=1257$ 794) of the surveyed exons, although we were unable to accurately survey the CNV status for eight exons included within the v2 panel (105 genes) due to consistently poor coverage across the cohort (online supplementary table S4). Consistently poor coverage was not observed across individuals surveyed through the newer v3 gene panel (180 genes; online supplementary table S4).

The average normalised NGS coverage profiles for each exon were calculated, and extensive variability was observed across the complete cohort, with average intersample variability values per exon of $21.1 \%$ ( $n$, exons $=313230$ ) and $22.2 \%$ (n, exons=954512) for the v2 and v3 gene panels, respectively (online supplementary figure S2). Intersample variation was reduced to $5.83 \%$ (n exons $=1224$ 686, median $=5.25 \%$, $\mathrm{SD}=3.28 \%$ ), when observations were limited to the extent of variation among samples selected as the reference set by ExomeDepth for each tested sample. There were 43056 exons excluded from this analysis due to the selection of a solitary sample as the reference set by ExomeDepth $(n=41512)$ or as a result of consistently poor coverage $(n=1544)$. In comparison to previously published simulation data sets, ${ }^{14} 95 \%$ and $99 \%$ of the surveyed exons are consistent with an accuracy for single exon deletions of $98.7 \%$ and $98.2 \%$, respectively (online supplementary figure S3).

\section{Confirmation of CNVs and clinical outcomes}

We confirmed 44/56 CNV events through orthogonal techniques, determining a positive predictive value (PPV) of $79 \%$ for the informatics filtering strategies employed in this study (figure 1, online supplementary results). Expanding confirmations to also include 13 events excluded through comparison to other CNV detection algorithms confirmed the presence of a single likely benign duplication event in NPHP1 (14016366; NM_000272.3:c.(?_-1)_("1_?)dup) but reduced the PPV to $65.2 \%(45 / 69)$. In confirming these findings, we determined a molecular diagnosis or a provisional molecular diagnosis for 25 individuals and additional findings that did not account for a molecular diagnosis for 18 individuals (table 1). These results were obtained after full appraisal of the clinical indication of IRD for the referred individual and the analysis of SNVs and small indels from routine gene panel NGS testing. Of note, a single individual was confirmed with two independent heterozygous CNV events, neither of which was determined to account for a molecular diagnosis (13009597; table 1). Routine testing identified a pathogenic missense variant in SNRNP200 (NM_014014.3: c.2042G>A, p.(Arg681His)), accounting for a diagnosis of autosomal dominant retinitis pigmentosa for this individual, with no pathogenic/likely pathogenic variants identified in-trans to the confirmed heterozygous deletion of $I D H 3 B$ and MKKS. Of the $25 \mathrm{CNVs}$ that enabled the confirmation or provisional confirmation of diagnosis, 23 confirmed autosomal recessive disorders and 2 confirmed autosomal dominant disorders (online supplemetary table S5). Twenty of these CNV events were confirmed in a heterozygous state, with 18 of the events suspected $(n=8)$ or confirmed $(n=10)$ to be in-trans to a heterozygous and proven/potentially pathogenic SNV or indel confirmed within the same gene (online supplementary table S5). Confirmation of in-trans variants included 


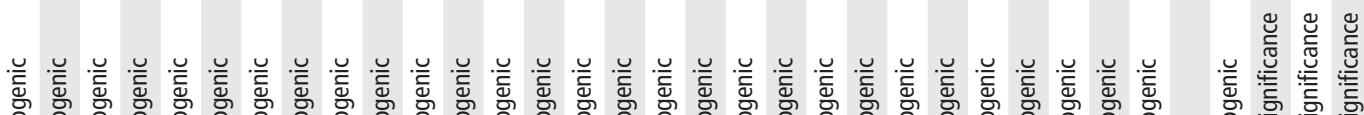
o

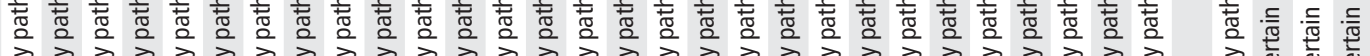

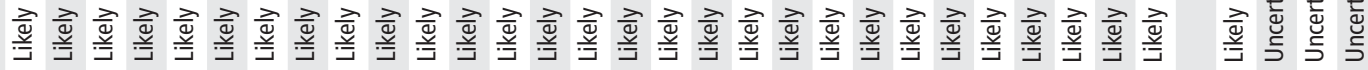
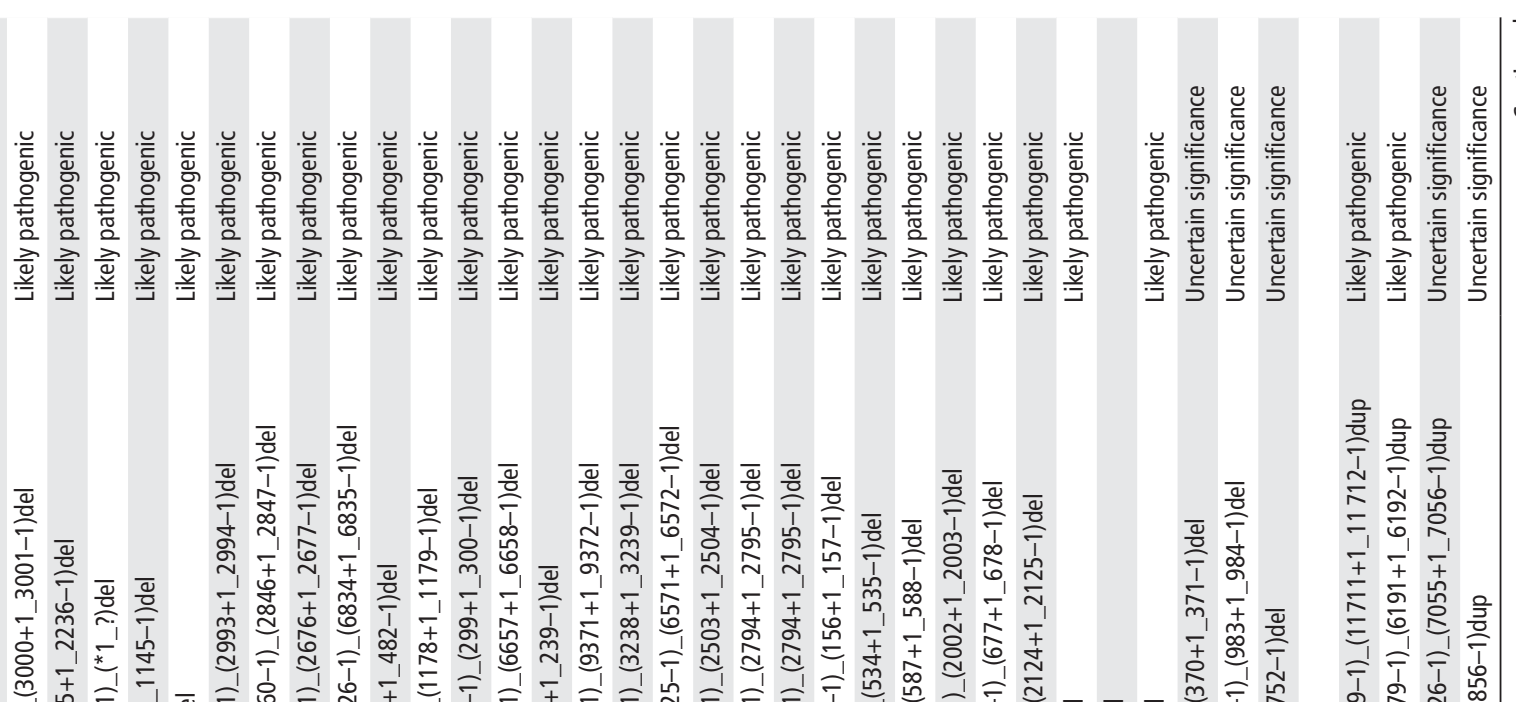

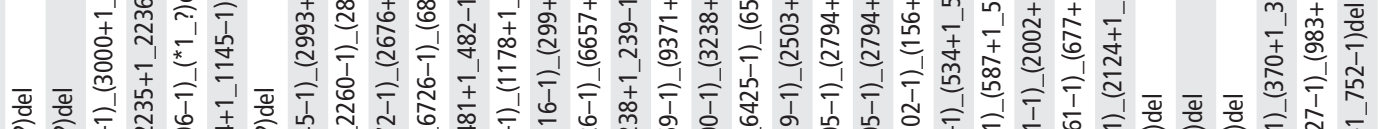

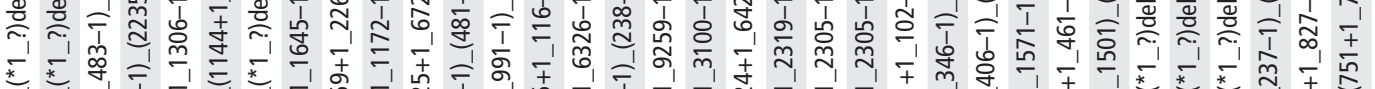

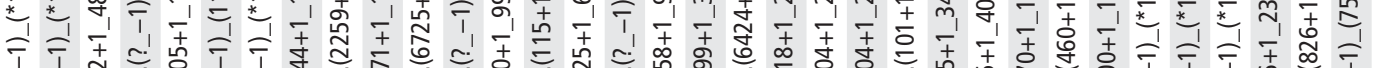
ป!

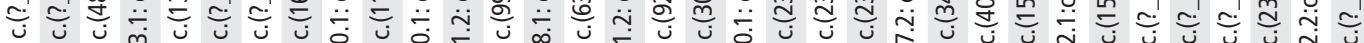

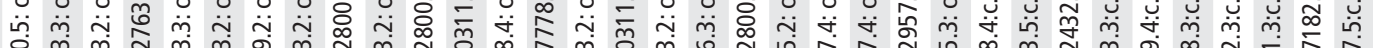

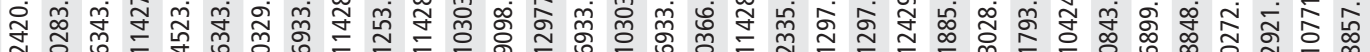

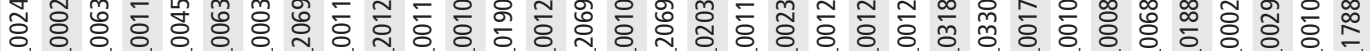

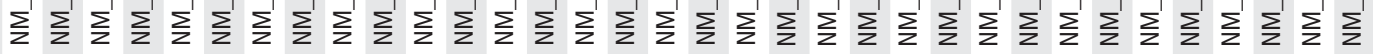

을 을 을

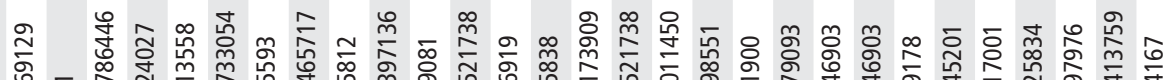

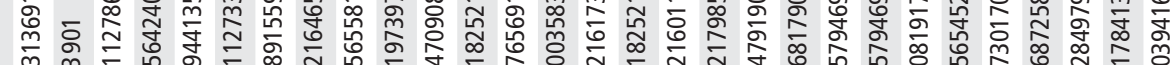

员

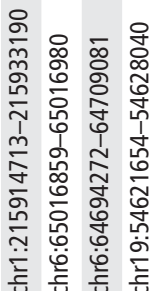

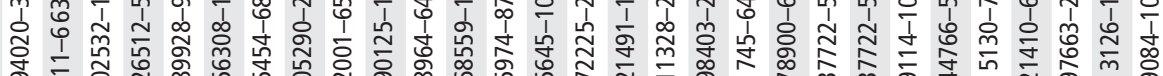

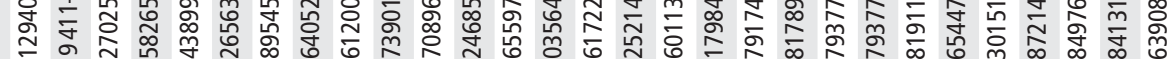

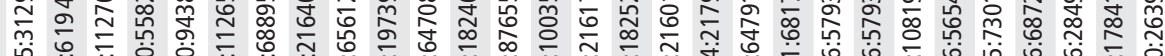

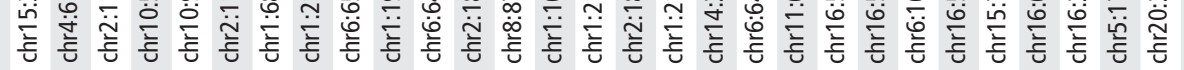

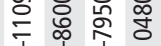

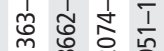

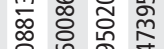

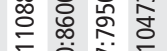

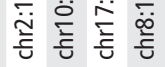

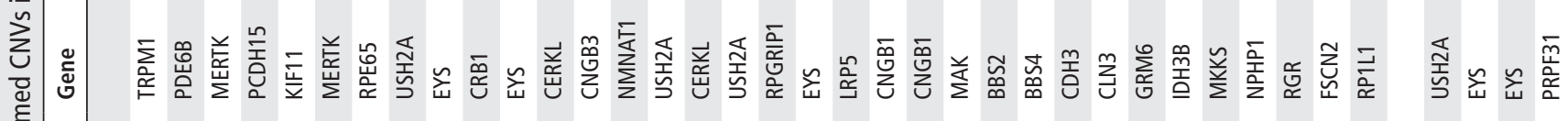




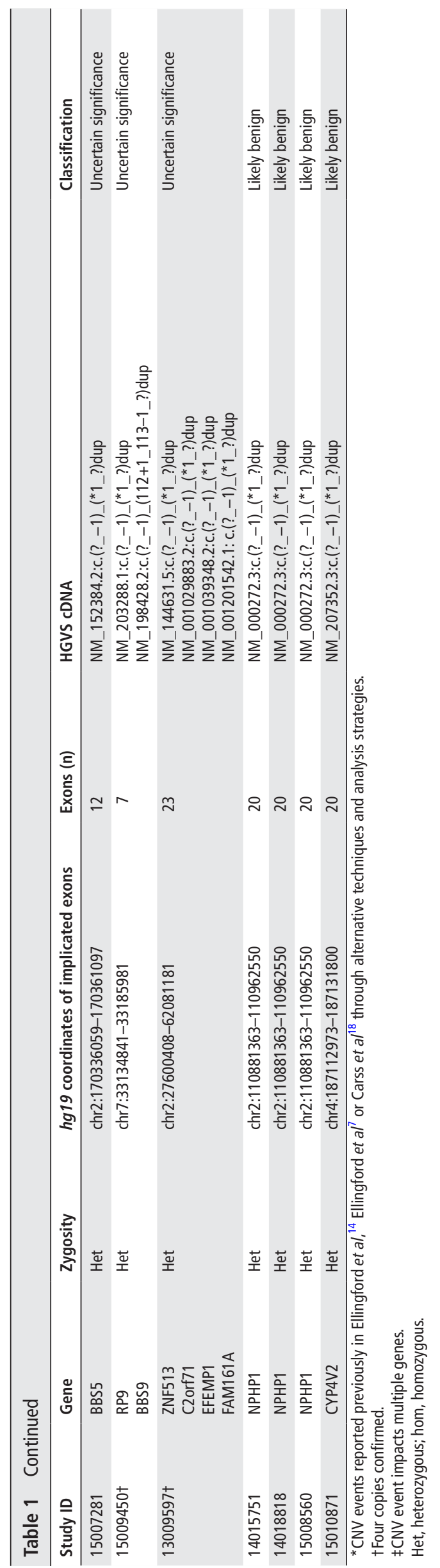

the encapsulation of an apparently homozygous SNV/indel by a heterozygous deletion event and/or familial segregation analysis. For example, a heterozygous whole gene deletion of RPE65 (NM_000329.2) was identified for an individual originally described with a clearly pathogenic homozygous missense variant (NM 000329.2: c.1102T>C, p.(Tyr368His)). Subsequent familial segregation analysis confirmed these events to be paternally and maternally inherited, respectively. Five homozygous $\mathrm{CNV}$ events were confirmed to account for a molecular diagnosis for referred individuals, including four homozygous deletions (table 1) and a single duplication event confirmed as four copies of EYS exons 34-35 (NM_001142800.1).

We confirmed that seven 'likely pathogenic' deletions were present in a carrier state, including two whole gene deletions, two deletions predicted to cause a frameshift and three inframe deletions. These events were all described in genes known as a cause of IRD or associated syndromic disorders that are inherited in an autosomal recessive manner, including BBS2, BBS4, CDH3, CLN3, GRM6, NPHP1, and a deletion spanning IDH3B and MKKS (table 1).

Duplications proved more complex for clinical interpretation, and based on current evidence most of the identified duplications were classified as 'uncertain significance' $(45 \%, \mathrm{n}=5)$ or to be 'likely benign' $(36 \%, n=4)$.

In four individuals, we identified heterozygous $\mathrm{CNV}$ events in genes known as a cause of autosomal dominant Mendelian disorders that were not determined to be a cause of disease for the referred individual (table 1). These included a threeexon deletion in RP1L1 (NM 178857.5), a single-exon deletion in FSCN2 (NM_001077182.2), a single-exon deletion in RGR (NM_002921.3) and a duplication event impacting RP9 (NM_203288.1) and BBS9 (NM_198428.2). Of note, we also identified four copies of PRPF $\overline{1}$ exons 2-8 (NM 015629.3) in an additional individual. Based on current evidence, the PRPF31 duplication was classified as 'uncertain significance' (online supplementary case study), although we expect future investigations to assist with the interpretation of this variant.

Population and in-house frequencies of identified CNV events To assist with clinical interpretation, the frequency of confirmed CNV events was determined through comparison to two independently acquired cohorts of WGS data sets generated for individuals with a clinical indication of IRD (605 through the NIHR BioResource Rare Diseases project using Illumina sequencing, and 77 through Complete Genomics sequencing). Of the 44 confirmed CNV events reported in this study, 25 (57\%) were found to have an overlap with events identified through WGS. This analysis was restricted to events identified through WGS, which overlapped at least $50 \%$ of the event identified through gene panel NGS. Three of these samples with identified CNV events were also included in the WGS cohorts (two from Illumina sequencing and one from Complete Genomics sequencing), enabling an assessment of the relative advantages for detecting CNVs through WGS in comparison to gene panel NGS (online supplementary figures S4,S5 and table 6) (should be table S6).Seven events were identified to have an overlap with more than one individual within the WGS cohorts (table 2). Of note, a confirmed duplication of RP9/BBS9 was identified in four unrelated WGS samples through Illumina sequencing (online supplementary figure S5). This information, in complement to other confirmed SNVs/indels for these individuals, permitted the classification of this duplication event as 'uncertain significance' and unlikely to account for the individual's molecular 
Table 2 CNVs identified in more than one unrelated individual

\begin{tabular}{|c|c|c|c|c|c|}
\hline \multirow[b]{2}{*}{ CNV event } & \multirow[t]{2}{*}{ CNV type } & \multicolumn{4}{|c|}{ Individuals (n) } \\
\hline & & Total & $\begin{array}{l}\text { Gene } \\
\text { panel NGS, } \\
n=550\end{array}$ & $\begin{array}{l}\text { WGS } \\
\text { (Illumina) } \\
n=605\end{array}$ & $\begin{array}{l}\text { WGS } \\
\text { (Complete } \\
\text { Genomics) } \\
n=77\end{array}$ \\
\hline CLN3 ex8-9 & Del & 6 & 1 & 5 & 0 \\
\hline MERTK ex1-7 & Del & 3 & 1 & 1 & 1 \\
\hline $\begin{array}{l}\text { NPHP1 (whole } \\
\text { gene) }\end{array}$ & Del/Dup & $7 / 10$ & $1 / 3$ & $5 / 6$ & $1 / 1$ \\
\hline RP9/BBS9 & Dup & 5 & 1 & 4 & 0 \\
\hline CNGB3 ex9-10 & Del & 4 & 1 & $3^{*}$ & 0 \\
\hline $\begin{array}{l}\text { CYP4V2 (whole } \\
\text { gene) }\end{array}$ & Dup & 5 & 1 & 4 & 0 \\
\hline $\begin{array}{l}\text { *Indicated, but u } \\
\text { ex8-10 for one in } \\
\text { Del, deletions; du } \\
\text { genome sequenc }\end{array}$ & $\begin{array}{l}\text { firmed, as } \\
\text { dual using } \\
\text { uplications }\end{array}$ & $\begin{array}{l}G B^{3} \\
\text { Car }\end{array}$ & $\begin{array}{l}0 \text { for two in } \\
\text { ad-depth alc } \\
\text { neration sec }\end{array}$ & $\begin{array}{l}\text { dividuals, a } \\
\text { orithm. } \\
\text { juencing; V }\end{array}$ & $\begin{array}{l}\text { CNGB3 } \\
\text {, whole }\end{array}$ \\
\hline
\end{tabular}

diagnosis. Similarly, whole gene duplication events of NPHP1 and CYP4V2 were identified in multiple unrelated individuals across the cohorts, and the absence of a second disease-causing mutation in these genes in all reported cases suggests they may represent benign variation. Future investigations into the pathogenicity of whole gene duplication events will assist with interpretation and will provide greater clarity in clinical reporting. These investigations may consist of WGS and/or long-read NGS to better characterise the location and phase of duplications, and RNA-seq experiments to assess the effect of duplications on gene expression.

\section{DISCUSSION}

A variety of techniques exist for the identification of genomic CNVs, including MLPA, Q-PCR, genome-wide and customised array $\mathrm{CGH}$, and low-coverage genome-wide sequencing. ${ }^{11}$ The detection of CNVs from high-coverage NGS data provides the unique opportunity for the simultaneous analysis of novel disease-causing SNVs and small indels, a strategy that has proved extremely successful for the diagnosis of IRD. ${ }^{9}$ While a number of informatics techniques exist for the identification of CNVs from NGS data sets, ${ }^{28}$ gene panel NGS approaches are limited by the types of CNV detection algorithms which can be routinely applied. Here, we describe an implemented informatics strategy using read-depth algorithms for the identification of CNVs from gene panel NGS data sets for 550 individuals with IRD. Through these strategies, we have confirmed 33 deletions and 11 duplications (table 1), determining these findings to contribute to the molecular diagnosis or provisional molecular diagnosis of IRD for 25 individuals (online supplementary table S5).

This study provides the largest cohort, to date, for the assessment of the relative frequency of CNVs as a cause of IRD from targeted NGS data sets. Our group and others have estimated the contribution of CNVs in IRDs from smaller cohorts of individuals, including high-resolution array CGH approaches $(3.5 \%$, $\mathrm{n}=57),{ }^{16}$ gene panel NGS $(3.1 \%, \mathrm{n}=126 ; 1.1 \%, \mathrm{n}=89 ; 6.4 \%$, $\mathrm{n}=47),{ }^{19} 2930$ WES $(10 \%, \mathrm{n}=60)^{17}$ and WGS $(10.9 \%, \mathrm{n}=46$; $12.5 \%, \mathrm{n}=16) .{ }^{731}$ Here, we show that CNVs contribute to a molecular diagnosis of IRD in $4.5 \%$ of cases, and are found without contribution to a molecular diagnosis in a further $3.3 \%$ of cases. Altogether, we estimate that a CNV is present within IRD genes in at least 1 in 13 individuals presenting with IRD, and thereby provides a significant and essential component of the diagnostic assessment.
The incorporation of read-depth CNV detection algorithms into gene panel NGS diagnostic services for IRD provides a realistic and cost-effective opportunity for widespread incorporation of $\mathrm{CNV}$ analysis. However, false-negative assessments, false-positive discoveries, complexity with clinical interpretation and the size of events that can be detected all provide significant limitations to this approach. ${ }^{32}$ To overcome these challenges in this study, we compared the results from ExomeDepth with two other publicly available CNV detection algorithms with the capability to detect single-exon CNV events (CoNVex ${ }^{22}$ and $\mathrm{CoNVaDING}^{12}$ ) and used distinct strategies for $\mathrm{CNV}$ filtering to reduce the number of false-positive events analysed (figure 1). These filtering approaches provided a PPV of $79 \%$ $(44 / 56)$ and enabled the confirmation of events with a range of confidence scores calculated by the ExomeDepth algorithm $(\min =6.7, \max =424)$, including 11 single-exon deletions and one single-exon duplication. Furthermore, we assessed two key quality assurance parameters previously identified as key determinants of false-negative assessments through ExomeDepth: insufficient coverage and intersample variability. ${ }^{14}$ We identified that $99.2 \%$ of surveyed exons had appropriate sequencing coverage for CNV surveillance in tested samples and that $99 \%$ of exons were consistent with a $98.2 \%$ accuracy of ExomeDepth in comparison to 1000 previously reported simulated single-exon deletion events. ${ }^{14}$ Importantly, the frequency of CNVs reported for this cohort are concordant with a recent study that interrogated rare variants in 224 IRD-associated genes from WGS data sets for 605 individuals with IRD,${ }^{18}$ and these data provide additional support for the sensitivity of the methodologies applied to gene panel NGS data sets in this study.

We have described CNVs in 36 different genes. The genes most frequently identified with CNVs were EYS $(n=5)$, USH $2 A(n=4)$ and NPHP1 $(\mathrm{n}=4)$ (table 1). These data are in accordance with recent findings that have identified factors underpinning susceptibility of IRD genes to CNVs. ${ }^{33}$ Microhomology-mediated DNA repair mechanisms (eg, microhomology-mediated break-induced replication) have been proposed as a major contributor to the genesis of non-recurrent CNVs. ${ }^{33} 34$ Our data sets precluded a comprehensive assessment of CNV mechanisms. However, it is notable that we have observed small stretches of microhomology between proximal and distal genomic sequences at breakpoints for non-recurrent CNVs (online supplementary table S6). We have also identified several instances of a recurrent duplication and a recurrent deletion of the complete coding region of NPHP1 (NM_000272.3), which are expected to have arisen through non-allelic homologous recombination between segmental duplications flanking NPHP1. ${ }^{35}$ The deletion of NPHP1 has been frequently reported as a cause of autosomal recessive juvenile nephronophthisis and Senior-Loken syndrome. The emergence of long-read NGS techniques to study CNVs will likely assist in the comprehensive characterisation of structural variant breakpoints, the elucidation of CNV genesis mechanisms, and the existence of ancestral and susceptibility haplotypes for CNVs that impact IRD genes.

In total we confirmed $44 \mathrm{CNV}$ events through the described informatics strategies (figure 1), including 12 whole gene events, 6 events removing or duplicating the canonical start or end codon, and 26 intragenic events. These strategies validated the presence of $28 \%$ and $16 \%$ of the deletions and duplications originally identified by ExomeDepth, respectively (figure 1). While these data suggest that IRD genes are more susceptible to deletion than duplication, our observations may be a limitation of the approaches applied, as NGS read-depth CNV detection software has been shown to be less sensitive for small duplication 
events. ${ }^{36}$ Duplications also proved more challenging for clinical interpretation as we were unable to determine phase of apparently homozygous events or confirm the genomic location of duplicated sequences. Both of these identified challenges may be overcome by the application of split-read and discordant read-pair algorithms to WGS data sets. ${ }^{28}$ A duplication identified in PRPF31, confirmed to be two extra copies of exons $2-8$, proved particularly problematic for clinical interpretation (online supplementary case study). Recently, Ayuso et al identified that a heterozygous duplication in PRPF31, encompassing exons 2-5, significantly reduced gene expression of PRPF31 and underpinned clinical presentation of retinitis pigmentosa. ${ }^{37}$ These results are consistent with the haploinsufficient pathogenic mechanism of mutations in PRPF31 and other pre-mRNA splicing factor genes. ${ }^{38}$ However, mutations in PRPF31 are often reported with incomplete penetrance, ${ }^{38}$ and the patient identified with this duplication in our cohort also carried a homozygous variant in another gene surveyed through gene panel NGS that could account for their molecular diagnosis of IRD (online supplementary case study). Future assessments of the location of duplicated sequences and their effect on PRPF31 gene expression will assist with clinical interpretation and will be of great interest.

Interestingly, we also identified a number of genes that were absent from CNVs, including $A B C A 4$, one of the most prevalent causes of IRD and a gene commonly identified to be in a carrier state in tested individuals. While it is possible that sequencing data generated for $A B C A 4$ have characteristics that reduce the accuracy of the read-depth $\mathrm{CNV}$ detection techniques described here, none of the three applied algorithms identified deletions or duplications disrupting or encapsulating $A B C A 4$, the sequencing profile is consistent with accurate surveillance of CNVs (onlinesupplementary table S7), and these findings are consistent with the absence and rare occurrence of CNVs in $A B C A 4$ in studies using WGS and array CGH for CNV interrogation. ${ }^{18} 3940$

Taken together, we demonstrate that CNVs provide a significant contribution towards the onset of IRD. We show that readdepth algorithms applied to gene panel NGS data sets generated for individuals with IRD can identify deletion and duplication events ranging from single exons to multigene events, and provide compelling evidence for the routine incorporation of $\mathrm{CNV}$ analysis as a first-tier diagnostic test for individuals with IRD.

\author{
Author affiliations \\ ${ }^{1}$ Manchester Centre for Genomic Medicine, Manchester Academic Health Sciences \\ Centre, Manchester University NHS Foundation Trust, St Mary's Hospital, Manchester, \\ UK \\ ${ }^{2}$ Division of Evolution and Genomic Sciences, Neuroscience and Mental Health \\ Domain, School of Biological Sciences, Faculty of Biology, Medicine and Health, \\ University of Manchester, Manchester, UK \\ ${ }^{3}$ Department of Genetics, UCL Institute of Ophthalmology, London, UK \\ ${ }^{4}$ Congenica, Wellcome Genome Campus, Hinxton, Cambridge, UK \\ ${ }^{5}$ Department of Haematology, University of Cambridge NHS Blood and Transplant \\ Centre, Cambridge, UK \\ ${ }^{6}$ Department of NIHR BioResource - Rare Diseases, Cambridge University Hospitals \\ NHS Foundation Trust, Cambridge Biomedical Campus, Cambridge, UK \\ ${ }^{7}$ Department of Medical Genetics, Cambridge Institute for Medical Research, \\ University of Cambridge, Cambridge, UK \\ ${ }^{8}$ Moorfields Eye Hospital NHS Foundation Trust, London, UK
}

Correction notice This article has been corrected since it was published Online First. A typo has been corrected.

Acknowledgements We thank all patients, referring clinicians, clinical scientists and genetic counsellors involved in this study. This work made use of data from the NIHR BioResource - Rare Diseases project.
Contributors JME and GCMB designed and coordinated the study. All authors contributed genetic and/or phenotypic data. JME wrote the manuscript, and all authors provided important revisions and intellectual content.

Funding This work was supported by the Biotechnology and Biological Sciences Research Council Biotechnology and Biological Sciences Research Council [grant code BB/J014478/1], the Manchester Biomedical Research Centre, the National Institute for Health Research Biomedical Centre at Moorfields Eye Hospital and the UCL Institute of Ophthalmology, RP Fighting Blindness and Fight for Sight (RP Genome Project GR586), the Cambridge Biomedical Research Centre, and an independent research grant funded by the Manchester Academic Health Science Centre.

Competing interests $\mathrm{CT}$ is an employee of Congenica Ltd. All other authors declare no competing interests.

Patient consent Detail has been removed from this case description/these case descriptions to ensure anonymity. The editors and reviewers have seen the detailed information available and are satisfied that the information backs up the case the authors are making.

Ethics approval Greater Manchester West Research Ethics Committee.

Provenance and peer review Not commissioned; externally peer reviewed.

Open Access This is an Open Access article distributed in accordance with the terms of the Creative Commons Attribution (CC BY 4.0) license, which permits others to distribute, remix, adapt and build upon this work, for commercial use, provided the original work is properly cited. See: http://creativecommons.org/ licenses/by/4.0/

(C) Article author(s) (or their employer(s) unless otherwise stated in the text of the article) 2018. All rights reserved. No commercial use is permitted unless otherwise expressly granted.

\section{REFERENCES}

1 Wright AF, Chakarova CF, Abd El-Aziz MM, Bhattacharya SS. Photoreceptor degeneration: genetic and mechanistic dissection of a complex trait. Nat Rev Genet 2010;11:273-84

2 Berger W, Kloeckener-Gruissem B, Neidhardt J. The molecular basis of human retinal and vitreoretinal diseases. Prog Retin Eye Res 2010;29:335-75.

3 Liew G, Michaelides M, Bunce C. A comparison of the causes of blindness certifications in England and Wales in working age adults (16-64 years), 1999-2000 with 2009-2010. BMJ Open 2014:4:e004015.

4 Ellingford JM, Sergouniotis PI, Lennon R, Bhaskar S, Williams SG, Hillman KA, O'Sullivan J, Hall G, Ramsden SC, Lloyd IC, Woolf AS, Black GC. Pinpointing clinical diagnosis through whole exome sequencing to direct patient care: a case of SeniorLoken syndrome. Lancet 2015;385:1916.

5 Lee K, Garg S. Navigating the current landscape of clinical genetic testing for inherited retinal dystrophies. Genet Med 2015;17:245-52.

6 Lee H, Deignan JL, Dorrani N, Strom SP, Kantarci S, Quintero-Rivera F, Das K, Toy T, Harry B, Yourshaw M, Fox M, Fogel BL, Martinez-Agosto JA, Wong DA, Chang VY, Shieh PB, Palmer CG, Dipple KM, Grody WW, Vilain E, Nelson SF. Clinical exome sequencing for genetic identification of rare Mendelian disorders. JAMA 2014:312:1880.

7 Ellingford JM, Barton S, Bhaskar S, Williams SG, Sergouniotis PI, O'Sullivan J, Lamb JA, Perveen R, Hall G, Newman WG, Bishop PN, Roberts SA, Leach R, Tearle R, Bayliss S, Ramsden SC, Nemeth AH, Black GC. Whole genome sequencing increases molecular diagnostic yield compared with current diagnostic testing for inherited retinal disease. Ophthalmology 2016;123:1143-50.

8 Consugar MB, Navarro-Gomez D, Place EM, Bujakowska KM, Sousa ME, Fonseca-Kelly ZD, Taub DG, Janessian M, Wang DY, Au ED, Sims KB, Sweetser DA, Fulton AB, Liu Q, Wiggs IL, Gai X, Pierce EA. Panel-based genetic diagnostic testing for inherited eye diseases is highly accurate and reproducible, and more sensitive for variant detection, than exome sequencing. Genet Med 2015;17:253-61.

9 Ellingford JM, Barton S, Bhaskar S, O'Sullivan J, Williams SG, Lamb JA, Panda B, Sergouniotis PI, Gillespie RL, Daiger SP, Hall G, Gale T, Lloyd IC, Bishop PN, Ramsden SC, Black GC. Molecular findings from 537 individuals with inherited retinal disease. J Med Genet 2016:761-7.

10 Stenson PD, Mort M, Ball EV, Shaw K, Phillips A, Cooper DN. The human gene mutation database: building a comprehensive mutation repository for clinical and molecular genetics, diagnostic testing and personalized genomic medicine. Hum Genet 2014;133:1-9.

11 Alkan C, Coe BP, Eichler EE. Genome structural variation discovery and genotyping. Nat Rev Genet 2011:12:363-76.

12 Johansson LF, van Dijk F, de Boer EN, van Dijk-Bos KK, Jongbloed JD, van der Hout AH, Westers H, Sinke RJ, Swertz MA, Sijmons RH, Sikkema-Raddatz B. CoNVaDING: Single Exon Variation Detection in Targeted NGS Data. Hum Mutat 2016;37:457-64.

13 Pugh TJ, Amr SS, Bowser MJ, Gowrisankar S, Hynes E, Mahanta LM, Rehm HL, Funke $B$, Lebo MS. VisCap: inference and visualization of germ-line copy-number variants from targeted clinical sequencing data. Genet Med 2016;18:712-9. 
14 Ellingford JM, Campbell C, Barton S, Bhaskar S, Gupta S, Taylor RL, Sergouniotis PI, Horn B, Lamb JA, Michaelides M, Webster AR, Newman WG, Panda B, Ramsden SC, Black GC. Validation of copy number variation analysis for next-generation sequencing diagnostics. Eur J Hum Genet 2017;25:719-24.

15 Bujakowska KM, Fernandez-Godino R, Place E, Consugar M, Navarro-Gomez D, White J, Bedoukian EC, Zhu X, Xie HM, Gai X, Leroy BP, Pierce EA. Copy-number variation is an important contributor to the genetic causality of inherited retinal degenerations. Genet Med 2017:19:643-51.

16 Van Cauwenbergh C, Van Schil K, Cannoodt R, Bauwens M, Van Laethem T, De Jaegere S, Steyaert W, Sante T, Menten B, Leroy BP, Coppieters F, De Baere E. arrEYE: a customized platform for high-resolution copy number analysis of coding and noncoding regions of known and candidate retinal dystrophy genes and retinal noncoding RNAs. Genet Med 2017;19:457-66.

17 Khateb S, Hanany M, Khalaileh A, Beryozkin A, Meyer S, Abu-Diab A, Abu Turky F, Mizrahi-Meissonnier L, Lieberman S, Ben-Yosef T, Banin E, Sharon D. Identification of genomic deletions causing inherited retinal degenerations by coverage analysis of whole exome sequencing data. J Med Genet 2016;53:600-7.

18 Carss KJ, Arno G, Erwood M, Stephens J, Sanchis-Juan A, Hull S, Megy K, Grozeva D, Dewhurst E, Malka S, Plagnol V, Penkett C, Stirrups K, Rizzo R, Wright G, Josifova D, Bitner-Glindzicz M, Scott RH, Clement E, Allen L, Armstrong R, Brady AF, Carmichael J, Chitre M, Henderson RH, Hurst J, MacLaren RE, Murphy E, Paterson J, Rosser E, Thompson DA, Wakeling E, Ouwehand WH, Michaelides M, Moore AT, Webster AR, Raymond FL; NIHR-BioResource Rare Diseases Consortium. Comprehensive Rare Variant Analysis via Whole-Genome Sequencing to Determine the Molecular Pathology of Inherited Retinal Disease. Am J Hum Genet 2017;100:75-90.

19 Eisenberger T, Neuhaus C, Khan AO, Decker C, Preising MN, Friedburg C, Bieg A, Gliem M, Charbel Issa P, Holz FG, Baig SM, Hellenbroich Y, Galvez A, Platzer K, Wollnik B, Laddach N, Ghaffari SR, Rafati M, Botzenhart E, Tinschert S, Börger D, Bohring A, Schreml J, Körtge-Jung S, Schell-Apacik C, Bakur K, Al-Aama JY, Neuhann T, Herkenrath P, Nürnberg G, Nürnberg P, Davis JS, Gal A, Bergmann C, Lorenz B, Bolz HJ. Increasing the yield in targeted next-generation sequencing by implicating CNV analysis, non-coding exons and the overall variant load: the example of retinal dystrophies. PLoS One 2013;8:18:e78496.

20 Li H, Durbin R. Fast and accurate short read alignment with Burrows-Wheeler transform. Bioinformatics 2009;25:1754-60.

21 Plagnol V, Curtis J, Epstein M, Mok KY, Stebbings E, Grigoriadou S, Wood NW, Hambleton S, Burns SO, Thrasher AJ, Kumararatne D, Doffinger R, Nejentsev S. A robust model for read count data in exome sequencing experiments and implications for copy number variant calling. Bioinformatics 2012;28:2747-54.

22 Vijayarangakannan P. CoNVex: Copy Number Variation form Exomes. ftp://ftp.sanger. ac.uk/pub/users/pv1/CoNVex/Docs/CoNVex.pdf2013.

23 Richards S, Aziz N, Bale S, Bick D, Das S, Gastier-Foster J, Grody WW, Hegde M, Lyon E, Spector E, Voelkerding K, Rehm HL; ACMG Laboratory Quality Assurance Committee. Standards and guidelines for the interpretation of sequence variants: a joint consensus recommendation of the American College of Medical Genetics and Genomics and the Association for Molecular Pathology. Genet Med 2015;17:405-23.

24 Chen X, Schulz-Trieglaff O, Shaw R, Barnes B, Schlesinger F, Källberg M, Cox AJ, Kruglyak S, Saunders CT. Manta: rapid detection of structural variants and indels for germline and cancer sequencing applications. Bioinformatics 2016;32:1220-2.

25 Roller E, Ivakhno S, Lee S, Royce T, Tanner S. Canvas: versatile and scalable detection of copy number variants. Bioinformatics 2016;32:2375-7.

26 Drmanac R, Sparks AB, Callow MJ, Halpern AL, Burns NL, Kermani BG, Carnevali P, Nazarenko I, Nilsen GB, Yeung G, Dahl F, Fernandez A, Staker B, Pant KP, Baccash J, Borcherding AP, Brownley A, Cedeno R, Chen L, Chernikoff D, Cheung A, Chirita R, Curson B, Ebert JC, Hacker CR, Hartlage R, Hauser B, Huang S, Jiang Y, Karpinchyk V, Koenig M, Kong C, Landers T, Le C, Liu J, McBride CE, Morenzoni M, Morey RE, Mutch K, Perazich H, Perry K, Peters BA, Peterson J, Pethiyagoda CL, Pothuraju K, Richter C, Rosenbaum AM, Roy S, Shafto J, Sharanhovich U, Shannon KW, Sheppy CG, Sun M, Thakuria JV, Tran A, Vu D, Zaranek AW, Wu X, Drmanac S, Oliphant AR, Banyai WC, Martin B, Ballinger DG, Church GM, Reid CA. Human genome sequencing using unchained base reads on self-assembling DNA nanoarrays.

Science 2010;327:78-81.

27 Carnevali P, Baccash J, Halpern AL, Nazarenko I, Nilsen GB, Pant KP, Ebert JC, Brownley A, Morenzoni M, Karpinchyk V, Martin B, Ballinger DG, Drmanac R. Computational techniques for human genome resequencing using mated gapped reads. J Comput Biol 2012;19:279-92.

28 Pirooznia M, Goes FS, Zandi PP. Whole-genome CNV analysis: advances in computational approaches. Front Genet 2015;6:138.

29 Weisschuh N, Mayer AK, Strom TM, Kohl S, Glöckle N, Schubach M, Andreasson S, Bernd A, Birch DG, Hamel CP, Heckenlively JR, Jacobson SG, Kamme C, Kellner U, Kunstmann E, Maffei P, Reiff CM, Rohrschneider K, Rosenberg T, Rudolph G, Vámos $R$, Varsányi $B$, Weleber RG, Wissinger $B$. Mutation detection in patients with retinal dystrophies using targeted next gheneration sequencing. PLoS One 2016;11:e0145951.

30 Perez-Carro R, Corton M, Sánchez-Navarro I, Zurita O, Sanchez-Bolivar N, SánchezAlcudia R, Lelieveld SH, Aller E, Lopez-Martinez MA, López-Molina MI, Fernandez-San Jose P, Blanco-Kelly F, Riveiro-Alvarez R, Gilissen C, Millan JM, Avila-Fernandez A, Ayuso C. Panel-based NGS reveals novel pathogenic mutations in autosomal recessive retinitis pigmentosa. Sci Rep 2016;6:19531.

31 Nishiguchi KM, Tearle RG, Liu YP, Oh EC, Miyake N, Benaglio P, Harper S, KoskiniemiKuendig H, Venturini G, Sharon D, Koenekoop RK, Nakamura M, Kondo M, Ueno S, Yasuma TR, Beckmann JS, Ikegawa S, Matsumoto N, Terasaki H, Berson EL, Katsanis $\mathrm{N}$, Rivolta C. Whole genome sequencing in patients with retinitis pigmentosa reveals pathogenic DNA structural changes and NEK2 as a new disease gene. Proc Natl Acad SCiU SA 2013;110:16139-44.

32 Tan R, Wang Y, Kleinstein SE, Liu Y, Zhu X, Guo H, Jiang Q, Allen AS, Zhu M. An evaluation of copy number variation detection tools from whole-exome sequencing data. Hum Mutat 2014:35:899-907.

33 Van Schil K, Naessens S, Van de Sompele S, Carron M, Aslanidis A, Van Cauwenbergh C, Mayer AK, Van Heetvelde M, Bauwens M, Verdin H, Coppieters F, Greenberg ME, Yang MG, Karlstetter M, Langmann T, De Preter K, Kohl S, Cherry TJ, Leroy BP, De Baere $\mathrm{E}$. Mapping the genomic landscape of inherited retinal disease genes prioritizes genes prone to coding and noncoding copy-number variations. Genet Med 2017 (Epub ahead of print: 27 Jul 2017).

34 Carvalho CM, Lupski JR. Mechanisms underlying structural variant formation in genomic disorders. Nat Rev Genet 2016;17:224-38.

35 Yuan B, Liu P, Gupta A, Beck CR, Tejomurtula A, Campbell IM, Gambin T, Simmons AD, Withers MA, Harris RA, Rogers J, Schwartz DC, Lupski JR. Comparative Genomic Analyses of the Human NPHP1 Locus Reveal Complex Genomic Architecture and Its Regional Evolution in Primates. PLoS Genet 2015;11:e1005686.

36 Fowler A, Mahamdallie S, Ruark E, Seal S, Ramsay E, Clarke M, Uddin I, Wylie H, Strydom A, Lunter G, Rahman N. Accurate clinical detection of exon copy number variants in a targeted NGS panel using DECoN. Wellcome Open Res; $1: 20$

37 Martin-Merida I, Sanchez-Alcudia R, Fernandez-San Jose P, Blanco-Kelly F, Perez-Carro R, Rodriguez-Jacy da Silva L, Almoguera B, Garcia-Sandoval B, Lopez-Molina MI, AvilaFernandez A, Carballo M, Corton M, Ayuso C. Analysis of the PRPF31 Gene in Spanish Autosomal Dominant Retinitis Pigmentosa Patients: A Novel Genomic Rearrangement. Invest Ophthalmol Vis Sci 2017:58:1045-53.

38 Rose AM, Bhattacharya SS. Variant haploinsufficiency and phenotypic non-penetrance in PRPF31-associated retinitis pigmentosa. Clin Genet 2016;90:118-26.

39 Zaneveld J, Siddiqui S, Li H, Wang X, Wang H, Wang K, Li H, Ren H, Lopez I, Dorfman A, Khan A, Wang F, Salvo J, Gelowani V, Li Y, Sui R, Koenekoop R, Chen R. Comprehensive analysis of patients with Stargardt macular dystrophy reveals new genotype-phenotype correlations and unexpected diagnostic revisions. Genet Med 2015:17:262-70

40 Zernant J, Xie YA, Ayuso C, Riveiro-Alvarez R, Lopez-Martinez MA, Simonelli F, Testa F, Gorin MB, Strom SP, Bertelsen M, Rosenberg T, Boone PM, Yuan B, Ayyagari R, Nagy PL, Tsang SH, Gouras P, Collison FT, Lupski JR, Fishman GA, Allikmets R. Analysis of the ABCA4 genomic locus in Stargardt disease. Hum Mol Genet 2014;23:6797-806. 